\title{
Spinal cord injury and labour: is epidural anaesthesia the best option?
}

\author{
Inês Delgado; ${ }^{1}$ Francisco Gouveia ${ }^{2}$; Inês Aguiar ${ }^{2}$; Fernando Pousa ${ }^{1}$; Ana Milheiro ${ }^{1}$ \\ ${ }^{1}$ Attending Anaesthesiologist, Centro Hospitalar Vila Nova de Gaia/Espinho, Porto, Portugal \\ ${ }^{2}$ Resident of Anaesthesiology, Centro Hospitalar Vila Nova de Gaia/Espinho, Porto, Portugal
}

\section{Background}

Physiologic manifestations of spinal cord injury (SCl) can complicate management during labour and delivery.

Autonomic dysreflexia (AD) is an imbalanced reflex sympathetic discharge in patients with $\mathrm{SCl}$ at or above the level of splanchnic sympathetic outflow, leading to potentially life-threatening hypertension and vasoconstriction ${ }^{[1]}$.

\section{Case Report}

\section{Pre-anaesthetic period}

- 28-year-old female with past history of traumatic dorsal SCl American Spinal Injury Association Grade B

- Loss of motor function below T9 and sparing of sensory function

- Antispasmodic chronic medication was suspended soon in the first trimester of pregnancy

- Uneventful pregnancy.

\section{Anaesthetic period}

- Informed consent was obtained

- ASA Standard and cardiotocography monitoring was established

- Epidural technique was permormed in L3-L4 space, midline approach, no complications

- Administration of bolus of $16 \mathrm{mg}$ ropivacaine $2 \%$ followed by perfusion $(8-12 \mathrm{mg} / \mathrm{h}$ )

- Adequacy of analgesia was closely monitored by assessing haemodynamic status and pain associated with uterine contractions.

\section{Labour progression and delivery}

- Labour progressed uneventfully culminating in an eutocic delivery of a healthy new-born.

\section{Post-anaesthetic period}

- Epidural catheter was withdrawn soon after episiorrhaphy

- Conventional analgesia protocol was established

- She remained haemodynamically stable and comfortable until discharge.

\section{Discussion}

Evidence recommends adequate analgesia in women with $\mathrm{SCl}$ during labour and delivery even if they lack sensation.

Neuraxial anaesthesia has proven efficacy for preventing $A D$. Its mechanism resides upon blocking afferent stimuli that arise from pelvic organs triggered by surgical stimulation and manual manipulation of the uterus.

Technical difficulties may arise as a result of kyphoscoliosis, previous spinal surgery and difficult positioning due to spasms and bony deformities.

The height of neuraxial block may also be unpredictable and impossible to evaluate.

Alternatives to epidural block must be considered in the case of patient refusal, contraindication or unsuccessful technique.

\section{Learning Points}

$A D$ and muscular rigidity associated with previous $\mathrm{SCl}$ may be successfully managed with epidural anaesthesia.

\section{References}

[1] Sharif $\mathrm{H}$, Hou S. Autonomic dysreflexia: a cardiovascular disorder following spinal cord injury, Neural (1) 